\title{
Optical observations of five X-ray selected BL Lacertae objects ${ }^{\star}$
}

\author{
M. Villata ${ }^{1}$, C.M. Raiteri ${ }^{1}$, M.D. Popescu ${ }^{2}$, G. Sobrito ${ }^{1,3}$, G. De Francesco ${ }^{1}$, L. Lanteri $^{1}$, and L. Ostorero ${ }^{3}$ \\ 1 Osservatorio Astronomico di Torino, Strada Osservatorio 20, I-10025 Pino Torinese, Italy \\ 2 Astronomical Institute of the Romanian Academy, St. Cutitul de Argint 5, 75212 Bucharest 28, Romania \\ 3 Dipartimento di Fisica Generale, Università di Torino, Via P. Giuria 1, I-10125 Torino, Italy
}

Received February 15; accepted April 11, 2000

\begin{abstract}
We present optical data obtained with the $1.05 \mathrm{~m}$ telescope of the Torino Astronomical Observatory for five X-ray selected BL Lacertae objects. The data are in the Johnson's $B, V$, and Cousins' $R$ bands. As the observing periods include the pointings of the Satellite per Astronomia X "Beppo" (BeppoSAX), our optical information will be comparable with the X-ray observations for a better understanding of the properties of these objects. The present data also provide optical information on sources that have been rarely observed in the optical band. Variability on short time scales (a few days) was found only for 1ES 1959+650.
\end{abstract}

Key words: BL Lacertae objects: general — BL Lacertae objects: individual: 1ES 0145+138, 1ES 0323+022, 1ES $1028+511,1 \mathrm{ES} 1118+424,1 \mathrm{ES} 1959+650$

\section{Introduction}

BL Lacertae objects (BL Lacs) are an extreme form of active galactic nuclei (AGNs) characterized by rapid variability, high polarization, a compact radio structure, and weak or absent emission lines. As BL Lacs have numerous characteristics in common with flat-spectrum radio quasars (FSRQs), they are classified together as "blazars". Their traits are generally interpreted as being the signature of a relativistic jet pointing towards us. For a better understanding of the phenomenon that creates such extreme features, we need to discriminate among the theoretical models that try to explain them. To make this study possible, a big observational effort is required. Optical observations offer a wealth of information on the

Send offprint requests to: M. Villata

* Tables 2-6 are only available in electronic form at the CDS via anonymous ftp to cdsarc.u-strasbg.fr (130.79.128.5) or via http://cdsweb.u-strasbg.fr/Abstract.html Correspondence to: villata@to.astro.it variability of BL Lacs. From such data, physical conditions of the emission region can be inferred.

As many important answers about the mechanisms that produce the emission at the various wavelengths are still to be given, simultaneous observations of these objects in different bands can help in making a better idea about what they really are, by revealing some possible correlations. The launch of satellites for astronomical researches made this kind of study possible. In particular, the collection of simultaneous optical and high-energy data is thought to be very important, since it allows to reconstruct that part of the spectral energy distribution (SED) of the source where a transition between the synchrotron emission and the inverse-Compton one is expected.

In Raiteri et al. (1998a) we presented optical data on eight X-ray selected BL Lacs observed in connection with pointings of BeppoSAX. The corresponding X-ray data were published in Wolter et al. (1998). In the following we present optical data for five X-ray selected BL Lacs observed in 1996-1999 in periods including the BeppoSAX observations of these sources.

\section{Observations and results}

The blazar optical monitoring program was started at the Torino Astronomical Observatory in November 1994 (e.g. Villata et al. 1997; Raiteri et al. 1998b). Data are obtained with the $1.05 \mathrm{~m}$ REOSC telescope equipped with a $1242 \times 1152$ pixels CCD camera. The scale of the CCD is 0.47 arcsec per pixel. In order to get spectral information, we generally observe in the Johnson's $B, V$, and Cousins' $R$ bands. Flat-field frames are taken both at sunset and at dawn, when possible.

Data are reduced with the Robin procedure locally developed (Lanteri 1999). The bias level is estimated from the image overscan; no dark current correction is needed since the CCD is cooled with liquid nitrogen. The point spread function is fitted by means of a circular Gaussian, the background being subtracted by fitting it with an 
Table 1. List of the observed BL Lacs

\begin{tabular}{llll}
\hline Source & RA (2000) & Dec. (2000) & Field photometry \\
\hline 1ES 0145+138 & 014829.7 & +140218 & Torino, unpublished \\
1ES 0323+022 & 032614.0 & +022515 & Smith et al. 1991 \\
1ES 1028+511 & 103118.5 & +505336 & Villata et al. 1998 \\
1ES 1118+424 & 112048.1 & +421212 & Torino, unpublished \\
1ES 1959+650 & 195959.8 & +650855 & Villata et al. 1998 \\
\hline
\end{tabular}

inclined plane. The blazar magnitude and corresponding uncertainty are derived by comparison with two reference stars in the same field of the source.

The coordinates at the 2000 equinox of the observed BL Lacs are given in Table 1, together with the reference for the photometric calibration of the comparison stars. In two cases $(1 \mathrm{ES} 0145+138,1 \mathrm{ES} 1118+424)$ we adopted unpublished standard magnitudes derived from observations performed at the REOSC telescope during photometric nights. The photometric calibration procedure is described in Villata et al. (1998).

The results of our observations are presented in Tables 2-6 and Figs. 1-5. The figures show light curves in the $R$ band, which is the best sampled one. The BeppoSAX pointings are indicated. In the subsets to the figures we plot the deviation from the mean of the magnitude difference between the two reference stars chosen in the same field of the source. This gives information on the reliability of the reference stars for each frame. The magnitude error lies between 0.02 and $0.09 \mathrm{mag}$ and is obtained as the maximum between the value in the subset and a lower limit that takes into account the magnitude difference between the source and the reference stars.

\section{1. $1 E S 0145+138$}

1ES $0145+138$ is an X-ray selected BL Lac, discovered by the Einstein satellite Slew Survey (Perlman et al. 1996). For this object, only one magnitude estimate was found in the literature: $V=17.9$, reported by Perlman et al. (1996).

We collected 11 frames in the $R$ band and 8 frames in the $V$ band, from October 22, 1996 to December 22, 1998. As can be seen from Fig. 1 and Table 2, we have two observations in the $R$ band and one in the $V$ band very close to the BeppoSAX pointing of December 30, 1997 $(\mathrm{JD}-2449000=1813.1)$. During our observing period no significant variation of the magnitude was seen: the brightest value for the $R$ band is $17.17 \mathrm{mag}$, and $\Delta R=0.44$.

\section{2. $1 E S 0323+022$}

This is an X-ray source discovered in the all-sky survey of the HEAO 1 satellite (Doxsey et al. 1983); it was also detected by the Slew Survey (Perlman et al. 1996). The

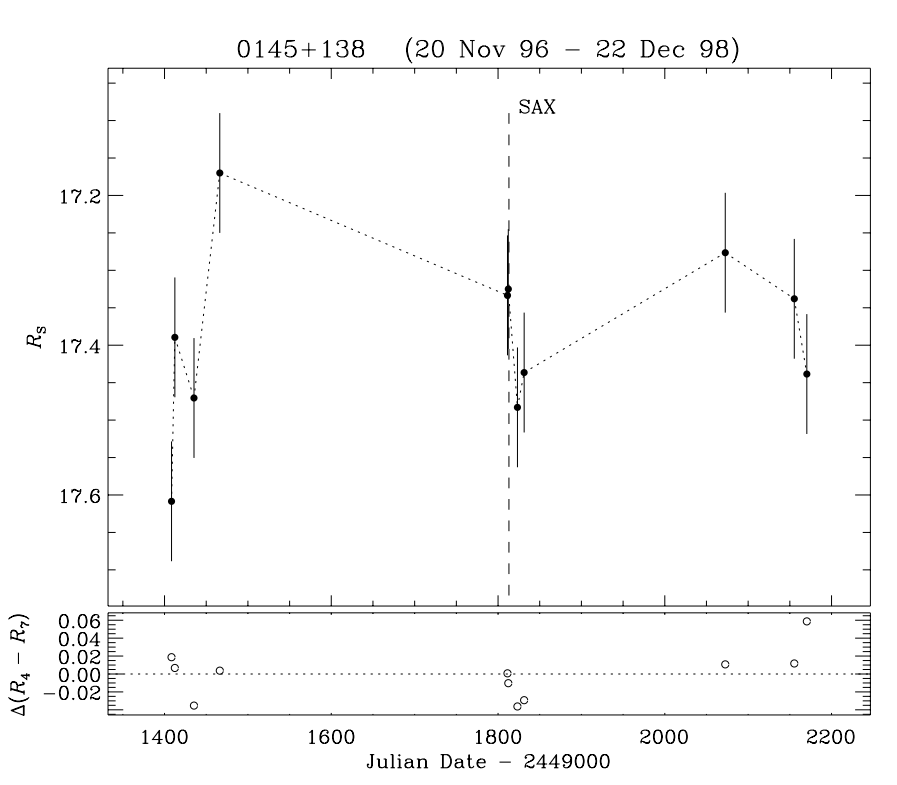

Fig. 1. Light curve of $1 \mathrm{ES} 0145+138$ in the $R$ band

first magnitude estimate of the optical counterpart was $V=16.5$ (Doxsey et al. 1983). Feigelson et al. (1986) obtained $V=16.55$ on November 28, 1983; observations made between October 14, 1982 and October 22, 1982 revealed a magnitude variation ( $V$ band) from 15.56 to $16.43 \mathrm{mag}$ and $B-V=0.41-0.96$; in December 1982, $V=16.08, B-V=0.59, V-R=0.46, R-I=0.52$; on January $1,1984, V=16.5$.

Feigelson et al. (1986) made an estimate of the brightness of the nebulosity surrounding $0323+022$ : they found $V=17.5$. They could also calculate the redshift and absolute magnitude of the host galaxy: $z=0.13 \pm 0.05$, $M_{V}=-21.6 \pm 0.7$.

Filippenko et al. (1986) reported large variations in the optical spectrum of $0323+022$ over a period of only 2 days, in late February 1985. They measured $V=17.2$ for the nucleus, 18.2 for the nebulosity, and $M_{V}=-21-22$ for the host galaxy.

Pica et al. (1988) reported seven measured magnitudes from September 1986 to January 1987, which indicate a maximum brightness of this object in that period of $B=17.45$, with variation $\Delta B=0.13$. 


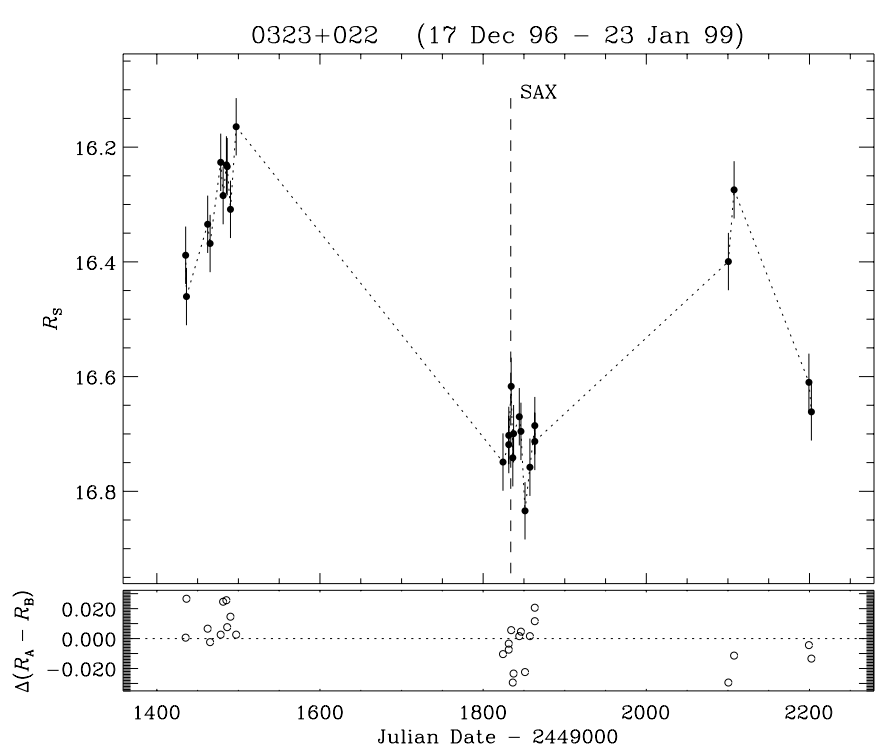

Fig. 2. Light curve of $1 \mathrm{ES} 0323+022$ in the $R$ band

Significant brightness variations were also reported by Jannuzi et al. (1993). In 101 nights of polarimetry and/or photometry observations during September 1987 March 1990, the following extreme values were recorded: $B=16.90-17.34, V=16.13-17.03, R=15.91-16.19$, $I=15.26-15.60$.

Fiorucci \& Tosti (1996) do not report any dramatic event in the optical monitoring program of this BL Lac. From February 1993 to January 1995 a mean value $\bar{V}=$ 16.70 was found, with $\Delta V=0.30$, and $\bar{I}=15.68$ with $\Delta I=0.32$; from October 1994 to January $1995, \bar{R}=$ 16.28, with $\Delta R=0.40(V-R=0.36, R-I=0.57)$.

Xie et al. (1996) reported $B V R I$ data taken in the period December 7, 1993 - December 31, 1994. No significant variations were detected: $B=17.44-17.52$, $V=16.78-16.92, R=16.15-16.41, I=15.76-15.84$.

Our dataset includes 26 frames in the $R$ band, 13 in the $V$ band, 9 in the $I$ band, and 3 in the $B$ band between October 23, 1996 and January 23, 1999 (see Table 3). The BeppoSAX pointing was scheduled for January 20, 1998 $(\mathrm{JD}-2449000=1833.8)$, a date around which we obtained a good sampling. In particular, we have $V R I$ data taken exactly in the same day of the BeppoSAX observation.

The light curve in the $R$ band, as can be seen in Fig. 2, shows an overall variation $\Delta R=0.67$, with a maximum brightness $R=16.16$.

\section{3. $1 E S 1028+511$}

This is a RASS and Slew Survey source for which very few photometric information exist. Only one value of the $V$ magnitude was found in the literature: $V=16.6$

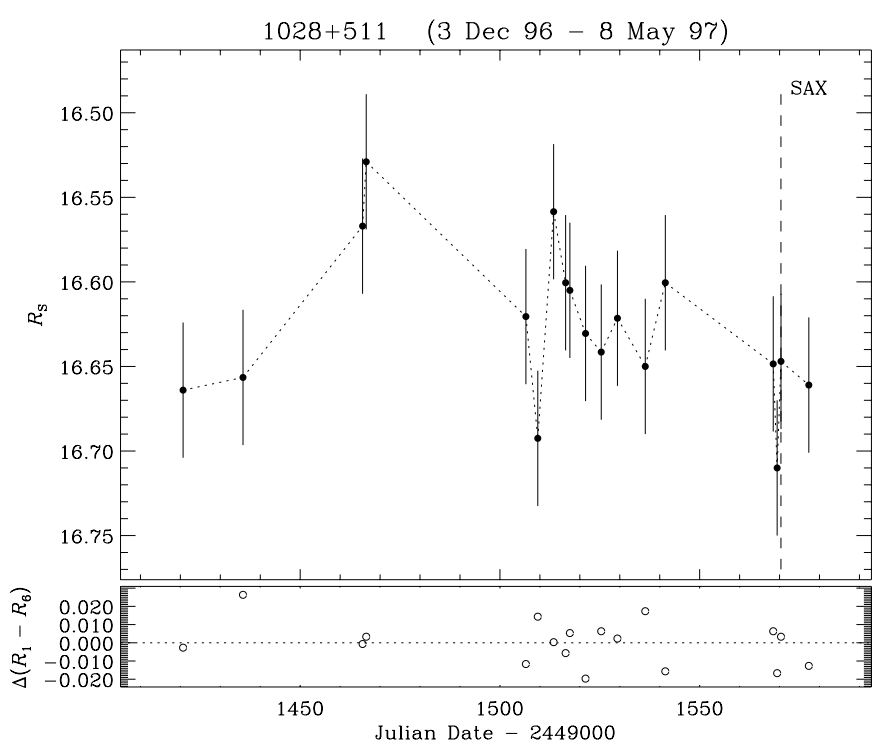

Fig. 3. Light curve of $1 \mathrm{ES} 1028+511$ in the $R$ band

(Perlman et al. 1996), and three values for the $B$ magnitude: $B=17.0$, derived from the spectrum based on an observation on May 29, 1992 (Schachter et al. 1993), $B=16.8$, from a spectrum taken between February and November 1992 (Bade et al. 1994), and $B=16.8$, as derived from HQS plates (Nass et al. 1996).

Our dataset regarding this object consists of 18 frames in the $R$ band, 2 in the $V$ band, and only one in the $B$ band, and covers the period December 3, 1996 - May 8, 1997 (see Table 4). BVR data were taken on May 1, 1997, simultaneously with the BeppoSAX pointing $(\mathrm{JD}-2449000=1570.4)$.

As seen in Fig. 3, a very thin variation of the magnitude was detected: $\Delta R=0.18$, and the maximum brightness was found at $R=16.53$.

\section{4. $1 E S 1118+424$}

This is an X-ray selected BL Lacertae object as was discovered by EXOSAT (and seen also with the Slew Survey).

Giommi et al. (1991) reported a $V$ magnitude of 17.3, and Perlman et al. (1996) $V=17.0$. The average brightness in the $R$ band in March 1993 was $R=18.0$ (Heidt \& Wagner 1998).

As can be seen in Fig. 4 and Table 5, we collected 11 data in the $R$ band between January 17 and May 21, 1997, and one of them is contemporaneous with the BeppoSAX observation of May 1, 1997 (JD-2449000 = 1570.1). The minimum value of the $R$ magnitude is 16.94 , and its variation is $\Delta R=0.32$. 


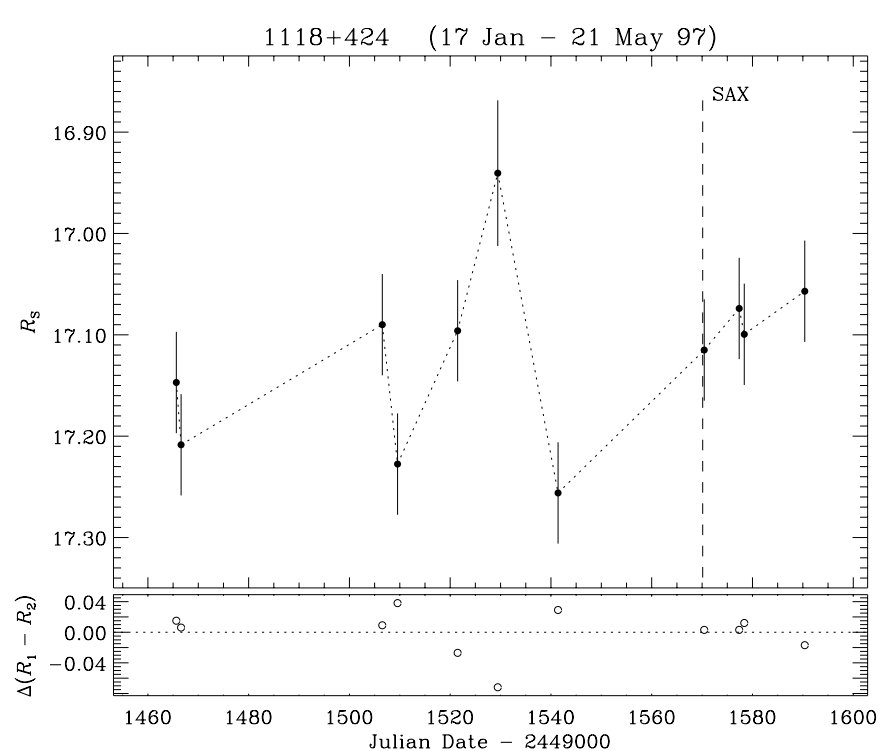

Fig. 4. Light curve of $1 \mathrm{ES} 1118+424$ in the $R$ band

\section{5. $1 E S 1959+650$}

It was identified in the $200 \mathrm{mJy}$ radio-selected sample and it was seen also in the Slew Survey. A detailed study of its host galaxy $\left(M_{R}=-23.0\right)$ was recently performed by Heidt et al. (1999), showing a complex structure possibly composed by an elliptical galaxy plus a disc and an absorption dust lane.

Three photometric optical values were found in the literature, showing a great variation of the source brightness in the optical band. It was reported as $V=16$ by Marcha (1994); $V=12.8$, as derived from digitalized plates, using $B-V=0.5$ (Schachter et al. 1993); and an approximate POSS O magnitude of 13.67 obtained from the Cambridge Automatic Plate Measurement (Perlman et al. 1996). More recently, Kurtanidze et al. (1999) report data taken in 1997-1999, with variations in the range $R=14.50-14.92$.

We observed this source from February 29, 1996 to May 30, 1997, and collected 16 frames in the $R$ band, 7 in the $V$ band, and 5 in the $B$ band (see Table 6). The BeppoSAX observation occurred on May 4, 1997 $(\mathrm{JD}-2449000=1573.4)$, near the end of our observing period. The light curve in the $R$ band is characterized by rapid flickering (see Fig. 5); in particular, a decrease of $0.28 \mathrm{mag}$ in 4 days was recorded on JD-2449000 = $1242.55-1246.55$.

This is the only object in the present sample showing rapid variability. Indeed, this BL Lac is also known as a radio source, so that it is not surprising that it presents the typical optical variability of radio-selected BL Lacs.

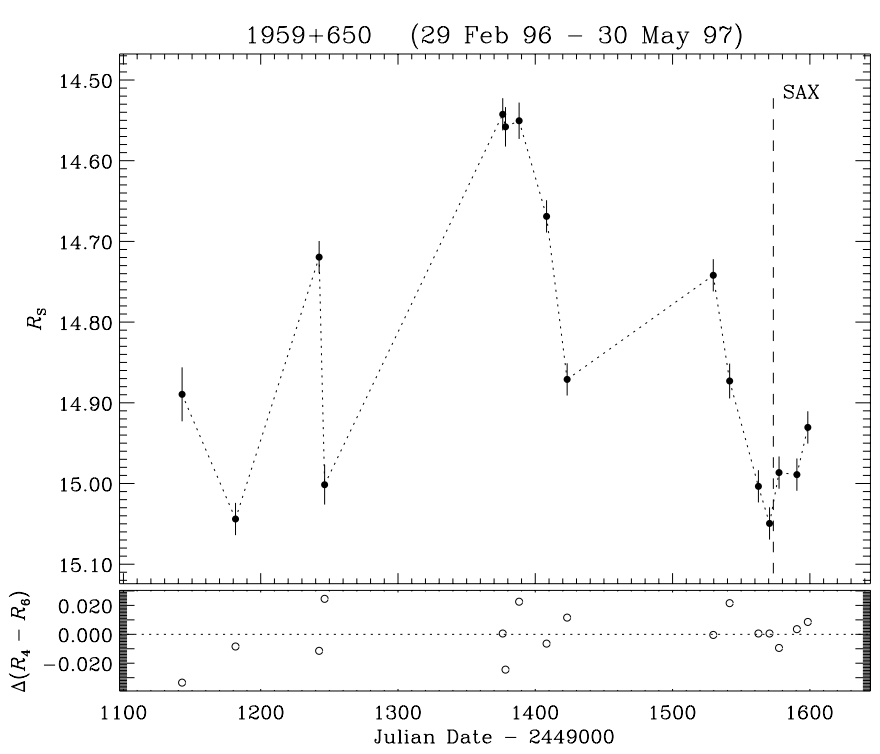

Fig. 5. Light curve of $1 \mathrm{ES} 1959+650$ in the $R$ band

Moreover, it has also been detected at $\mathrm{TeV}$ energies by the Utah Seven Telescope Array.

\section{Conclusions}

In this short paper we have reported optical data for five X-ray selected BL Lacertae objects. The data, taken with the $1.05 \mathrm{~m}$ telescope of the Torino Astronomical Observatory from February 29, 1996 to January 23, 1999, are in the Johnson's $B, V$, and Cousins' $R$ bands. In only one case (1ES $1959+650)$ variability on a time scale of a few days was observed.

The data presented in this paper represent an important contribution to the study of these objects, as optical observation of them was seldom performed in the past. All of our observation periods include pointings of the BeppoSAX satellite, and in some cases our observations are strictly simultaneous with them. This will allow an easy comparison of our optical data with the X-ray ones and also with those at other wavelengths, if available. In this way, information on the emission processes of the BL Lacs jets can be derived.

Our data confirm that generally the X-ray selected BL Lacs do not present strong optical variability, in comparison with the radio selected ones.

Acknowledgements. This work was partly supported by the Italian Ministry for University and Research (MURST) under grant Cofin98-02-32 and by the Italian Space Agency (ASI). 


\section{References}

Bade N., Fink H.H., Engels D., 1994, A\&A 286, 381

Doxsey R., Bradt H., McClintock J., et al., 1983, ApJ 264, L43

Feigelson E.D., Bradt H., McClintock J., et al., 1986, ApJ 302, 337

Filippenko A.V., Djorgovski S., Spinrad H., Sargent W.L.W., 1986, AJ 91, 49

Fiorucci M., Tosti G., 1996, A\&AS 117, 475

Giommi P., Tagliaferri G., Beuermann K., et al., 1991, ApJ 378,77

Heidt J., Wagner S.J., 1998, A\&A 329, 853

Heidt J., Nilsson K., Sillanpää A., Takalo L.O., Pursimo T., 1999, A\&A 341, 683

Jannuzi B.T., Smith P.S., Elston R., 1993, ApJS 85, 265

Kurtanidze O.M., Richter G.M., Nikolashvili M.G., 1999, in: Raiteri C.M., Villata M., Takalo L.O. (eds.) Proc. OJ94 Annual Meeting 1999, Blazar Monitoring towards the Third Millennium, Osservatorio Astronomico di Torino, Pino Torinese, p. 29

Lanteri L., 1999, in: Raiteri C.M., Villata M., Takalo L.O. (eds.) Proc. OJ-94 Annual Meeting 1999, Blazar
Monitoring towards the Third Millennium, Osservatorio Astronomico di Torino, Pino Torinese, p. 125

Marcha M., 1994, D. Sc. Thesis, Univ. Manchester

Nass P., Bade N., Kollgaard R.I., et al., 1996, A\&A 309, 419

Perlman E.S., Stocke J.T., Schachter J.F., et al., 1996, ApJS 104,251

Pica A.J., Smith A.G., Webb J.R., et al., 1988, AJ 96, 1215

Raiteri C.M., Villata M., De Francesco G., et al., 1998a, A\&AS 132,361

Raiteri C.M., Ghisellini G., Villata M., et al., 1998b, A\&AS 127,445

Schachter J.F., Stocke J.T., Perlman E., et al., 1993, ApJ 412, 541

Smith P.S., Jannuzi B.T., Elston R., 1991, ApJS 77, 67

Villata M., Raiteri C.M., Ghisellini G., et al., 1997, A\&AS 121, 119

Villata M., Raiteri C.M., Lanteri L., Sobrito G., Cavallone M., 1998, A\&AS 130, 305

Wolter A., Comastri A., Ghisellini G., et al., 1998, A\&A 335, 899

Xie G.Z., Zhang Y.H., Li K.H., et al., 1996, AJ 111, 1065 